CONFORMAL GEOMETRY AND DYNAMICS

An Electronic Journal of the American Mathematical Society

Volume 4, Pages 1-21 (February 23, 2000)

S $1088-4173(00) 00044-8$

\title{
UNBOUNDED COMPONENTS IN PARAMETER SPACE OF RATIONAL MAPS
}

PETER M. MAKIENKO

\begin{abstract}
Using pinching deformations of Riemann surfaces, we give several sufficient criteria for the space of quasiconformal deformations of rational map $R$ of degree $d$ to have non-compact closure in the space $R a t_{d}$ of rational maps of degree $d$ modulo conjugation by Möbius transformations.
\end{abstract}

\section{INTRODUCTION}

Let $d>1$ be an integer. Then the space of rational maps of degree $d$ may be identified with an open subset of $\mathbb{C} P^{2 d+1}$ via

$$
R(z)=\frac{a_{d} z^{d}+\cdots+a_{0}}{b_{d} z^{d}+\cdots+b_{0}} \rightarrow\left[a_{d}: \cdots: a_{0}: b_{d}: \cdots: b_{0}\right] \in \mathbb{C} P^{2 d+1}-V(\Delta),
$$

where $V(\Delta)$ is the locus where the resultant of numerator and denominator vanishes. The group $P S L_{2}(\mathbb{C})$ of Möbius transformations acts on this space by conjugation, yielding a Hausdorff quotient space $R_{a} t_{d}$ whose elements represent Möbius conjugacy classes of rational maps degree $d$. In general, if $R_{n} \rightarrow R$ in $R a t_{d}$, the dynamics of the limiting map $R$ may be quite different from that of the $R_{n}$-consider e.g. the complicated behavior in the quadratic family $z^{2}+c$. However, by work of MSS and [L], one knows that there is an open dense subset $W \subset \operatorname{Rat}_{d}$ consisting of structurally stable maps such that if $R_{n}, R \in W$ and $R_{n} \rightarrow R$, then the dynamics of $R_{n}$ converge to that $R$ in the sense that there exist quasiconformal conjugacies $h_{n}$ conjugating $R$ to $R_{n}$ such that $h_{n} \rightarrow i d$. Thus, changes in the dynamics can occur only on $\partial W$.

In this case when a sequence $R_{n}$ is "unbounded", the limiting dynamical system $R$ does not necessarily exist. As an example, consider $z^{2}+c_{n}$ with $c_{n} \rightarrow \infty$. For $n$ large enough the maps lie in a single component of $W$ (the complement of the Mandelbrot set) but no limiting quadratic map exists, regardless of how the maps are normalized.

In this paper, we study the question of unboundedness of certain kinds of deformation spaces associated with rational maps.

Received by the editors December 27, 1998 and, in revised form, September 14, 1999.

2000 Mathematics Subject Classification. Primary 37F45; Secondary 37F30.

This work has been partially supported by the Russian Fund of Basic Researches, Grant 9901-01006.

(C)2000 American Mathematical Society 
Definition 0.1. Let $R \in \mathbb{C} P^{2 d+1}$ be a rational map. The component of $\mathbf{J}$-stability of $R$ is the following space.

$q c_{J}(R)=\left\{F \in \mathbb{C} P^{2 d+1}:\right.$ there are neighborhoods $U_{R}$ and $U_{F}$ of $\mathbf{J}(R)$ and $\mathbf{J}(F)$, respectively and a quasiconformal homeomorphism $h_{F}: U_{R} \rightarrow U_{F}$ such that $\left.F=h_{F} \circ R \circ h_{F}^{-1}\right\} / P S L_{2}(\mathbb{C})$.

If $q c_{J}(R)$ has non-compact closure in $R a t_{d}$, we say that $q c_{J}(R)$ is unbounded. Equivalently, $q c_{J}(R)$ is unbounded if there is a sequence of Möbius conjugacy classes in $q c_{J}(R)$ such that any corresponding sequence of representatives in $\mathbb{C} P^{2 d+1}-V(\Delta)$ leaves every compact subset.

If $R$ is hyperbolic (i.e. every critical point converges to an attracting cycle), then $q c_{J}(R)$ is known as the hyperbolic component containing $R$.

Definition 0.2. Let $R \in \mathbb{C} P^{2 d+1}$ be a rational map. The space of quasiconformal deformations is defined as:

$q c(R)=\left\{F \in \mathbb{C} P^{2 d+1}:\right.$ there is a quasiconformal automorphism $h_{F}$ of the Riemann sphere $\overline{\mathbb{C}}$ such that $\left.F=h_{F} \circ R \circ h_{F}^{-1}\right\} / P S L_{2}(\mathbb{C})$.

The subspace $q c(R)$ is an everywhere dense subset of $q c_{J}(R)$; the complement consists of maps whose dynamics near the Julia set is essentially the same, but whose critical points in the Fatou set behave in an essentially different way. In this paper, we give conditions on the dynamics of a rational map $R$ which imply that the $J$-stable component of $R$ is unbounded (see Theorem $\mathrm{A}$ below). Theorem $\mathrm{A}$ is related to the following two problems:

1. (i) (C. McMullen [MM1]) If $R$ is a rational map of degree $d$ with connected Julia set and no common periodic point on the boundary of two components of the Fatou set, is the closure of the $J$-stable component of $R$ compact in Rat ${ }_{d}$ ?

Recently C. McMullen reformulated this problem (see [MM2]) as follows.

(ii) Let $R$ be a hyperbolic rational map. If the Julia set of $R$ is a Sierpinski carpet, is the closure of the $J$-stable component of $R$ compact in Rat R $_{d}$ ?

The Julia set $J(R)$ is a Sierpinski carpet means that $J(R)$ is obtained from the sphere by removing a countable dense set of open disks, bounded by disjoint Jordan curves whose diameters tend to zero.

2. (J. Milnor $[\mathrm{M})$ Let $d=2$. How can one decide whether a given hyperbolic component has compact closure in Rat R $_{2}$ or whether it is unbounded?

To state our main theorems, we briefly recall a description of these quotient Riemann surfaces, and the notion of an accessible point in the Julia set.

Quotient Riemann surfaces. Due to Sullivan $[\underline{S}$ there are only 5 types of periodic domains which can occur in the Fatou set for a rational map $R$. Furthermore there exists a Riemann surface associated with each type of these domains.

The cases with a fundamental domain:

(1) Let $D$ be an attractive periodic domain. Then the associated Riemann surface $\mathbf{T}_{D}$ is a torus with marked points $a_{1}, \ldots, a_{l}$, where $a_{i}$ correspond to those orbits of critical points of the full orbit of $D$ which do not land at the attractive 
cycle. Denote by $S_{D}$ the surface $\mathbf{T}_{D} \backslash\left\{a_{1}, \ldots, a_{l}\right\}$ and by $D_{R}$ the full orbit of $D$ minus the closure of the full orbit of the set of critical points. Then there is an unbranched covering

$$
P_{D}: D_{R} \rightarrow \mathbf{S}_{D}
$$

(2) Let $D$ be a parabolic periodic domain. Then the associated Riemann surface $\mathbf{S p}_{D}$ is a twice punctured sphere with marked points $a_{1}, \ldots, a_{l}$, where $a_{i}$ again correspond to the orbits of critical points of the full orbit of $D$. Denote by $S_{D}$ the surface $\mathbf{S p}_{D} \backslash\left\{a_{1}, \ldots, a_{l}\right\}$ and by $D_{R}$ the full orbit of $D$ minus the set of full orbits of critical points. Then there is an unbranched covering

$$
P_{D}: D_{R} \rightarrow \mathbf{S}_{D}
$$

Foliated cases:

(1) Let $D$ be a periodic Siegel disk of period $k$. Then associated Riemann surface $\mathbf{D}_{D}$ is the unit disk conformally isomorphic to $D$ with marked points $a_{1}, \ldots, a_{l+1}$ and is equipped with the cyclic group $\mathbf{G}_{D}$ generated by a rotation by angle $2 \pi i \alpha$, where $\alpha$ is irrational and is determined by the dynamics of $R$. Here the point $a_{1} \in \partial \mathbf{D}_{D}$. The points $a_{2}, \ldots, a_{l+1} \in \mathbf{D}_{D} \backslash \partial \mathbf{D}_{D}$ correspond to the first hits of forward orbits of the critical points into $D$. The group $\mathbf{G}_{D}$ corresponds to the action $R^{k}: D \rightarrow D$. Then there exists a branched covering $P_{D}$ from the full orbit of $D$ onto $\mathbf{D}_{D}$ semi-conjugating $R^{k}$ with the generator of $\mathbf{G}_{D}$. The covering $P_{D}$ is branched over the points $a_{2}, \ldots, a_{l+1}$. Denote by $S_{D}$ the surface $\mathbf{D}_{D} \backslash\left\{a_{2}, \ldots, a_{l+1}\right\}$.

(2) Let $D$ be a periodic Herman ring of period $k$. Then the associated Riemann surface $\mathbf{H}_{D}$ is a circular ring conformally equivalent to $D$ with marked points $a_{1}, \ldots, a_{l+2}$ and is equipped with the cyclic group $\mathbf{G}_{D}$ generated by rotation by angle $2 \pi i \alpha$, where $\alpha$ is irrational and is determined by the dynamics of $R$. Here the points $a_{1}$ and $a_{2}$ belong to different components of $\partial \mathbf{H}_{D}$. The points $a_{3}, \ldots, a_{l+2}$ correspond to the first hits of forward orbits of critical points. The group $\mathbf{G}_{D}$ corresponds to the action $R^{k}: D \rightarrow D$. Then there exists a branched covering $P_{D}$ from the full orbit of $D$ onto $\mathbf{H}_{D}$ semi-conjugating $R^{k}$ with the generator of $\mathbf{G}_{D}$. The covering $P_{D}$ is branched over the points $a_{3}, \ldots, a_{l+2}$. Denote by $S_{D}$ the surface $\mathbf{H}_{D} \backslash\left\{a_{3}, \ldots, a_{l+2}\right\}$.

(3) Let $D$ be a superattractive periodic domain of period $k$. Then the associated Riemann surface $S_{D}$ is a circular ring with marked points $a_{1}, \ldots, a_{l+2}$ and equipped with the group $\mathbf{G}_{D}$ generated by rotations by angles $2 \pi i \alpha, \alpha=$ $\frac{1}{\delta^{n}}, n=1,2, \ldots$, where $\delta$ is the local degree of $R^{k}$ at the superattractive point. Here points $a_{1}$ and $a_{2}$ belong to different components of $\partial S_{D}$ and all points $a_{1}, \ldots, a_{l+2}$ correspond to the forward orbits of critical points. In this case there is no map from the full orbit of $D$ onto $S_{D}$.

In the last three cases, the Riemann surface is foliated by concentric circles.

Denote by $S_{R}$ the disjoint union $\sqcup S_{D}$. Let $F_{R}$ be the Fatou set $\mathbf{F}(R)$ minus the full orbits of all superattractive periodic domains together with the closure of the full orbit of all critical points into $S_{R}$. Then there exists an unbranched covering $P_{R}: F_{R} \rightarrow S_{R}$.

\section{Accesses.}

Definition 0.3. Let $D \subset \overline{\mathbb{C}}$ be an open subset. Then a point $x \in \partial D$ is called accessible if there exists an arc (path) $\gamma \in D$ landing at $x$. 
Let $x \in \partial D$ be a periodic point accessible from $D$. Let $\omega_{1}$ and $\omega_{2} \subset D$ be two arcs (paths) landing at the point $x$. Then $\omega_{1}$ is equivalent to $\omega_{2}$ if these two paths are homotopic by a homotopy leaving the boundary of $D$ fixed. The equivalence class of an arc $\omega \subset D$ landing at the point $x \in \partial D$ is called an access to $x$ from $D$ and is denoted by $(x,[\omega])$.

Let $D \subset \mathbf{F}(R)$ be an attractive (parabolic) periodic domain of a given rational map $R$. Let $\gamma \subset S_{D}$ be a closed simple geodesic. Then a connected component $\beta \in P_{R}^{-1}(\gamma) \cap D$ is called a main component of the lift of $\gamma$ if $\beta$ lands at the attractive (parabolic) periodic point.

An access $(x,[\omega])$ is called geodesic if there is a geodesic $\gamma \subset \mathbf{S}_{D}$ and a main component $\beta$ of the lift of $\gamma$ such that $(x,[\beta])$ is equivalent to $(x,[\omega])$.

A periodic point $x \in \partial D$ is called geodesically accessible if $x$ admits a geodesic access from $D$.

We call two geodesic accesses $(x,[\beta])$ and $\left(y,\left[\beta_{1}\right]\right)$ independent if two geodesics $\gamma$ and $\gamma_{1} \subset S_{R}$ corresponding to these accesses are either mutually disjoint or coincide.

Main results. Consider the following sets of rational maps.

$\mathbf{W}_{1}=$ rational maps $R$ with disconnected Julia set $\}$

$\mathbf{W}_{2}=$ rational maps $R$ with connected Julia set such that there is a periodic component $D$ of the Fatou set and a periodic point $x \in \partial D$ having more than one access from $D$ \}

$\mathbf{W}_{3}=$ rational maps $R$ with connected Julia set having two periodic components $D_{1}$ and $D_{2}$ of the Fatou set such that the intersection $\partial D_{1} \cap \partial D_{2}$ contains two periodic points $x$ and $y$ accessible from both $D_{1}$ and $\left.D_{2}\right\}$

Theorem A. Let $R$ be a rational map of degree $d$.

(1) Let $R \in \mathbf{W}_{1}$. Then the component of $\mathbf{J}$-stability $q c_{J}(R)$ is unbounded.

(2) Let $R \in \mathbf{W}_{2} \cup \mathbf{W}_{3}$. Assume there is a map $R_{1} \in q c_{J}(R)$ such that the accesses in the definitions of $\mathbf{W}_{2}$ and $\mathbf{W}_{3}$ are geodesic and independent for $R_{1}$. Then $q c_{J}(R)$ is unbounded.

Corollary A. Let $R \in \mathbf{W}_{2} \cup \mathbf{W}_{3}$ be of degree $d$. Then there is an integer $N(R)$ such that $q c_{J}\left(R^{n}\right)$ is unbounded for all $n$ divisible by $N(R)$.

The following example shows that $N(R)$ can be greater than 1 .

Example A. Let $R$ be a rational map of degree $d$ with a completely invariant parabolic domain $D$. Assume that $\mathbf{J}(R)$ is connected and $\mathbf{S p}_{D}$ is a twice punctured sphere with only one marked point. Then the closure of the space $q c(R)$ is bounded in the space Rat $_{d}$. Moreover,

(1) if $\operatorname{deg}(R)=2$, then $q c_{J}(R)$ is bounded;

(2) if $\operatorname{deg}(R)=2$ and $R$ has an attractive periodic point of a period $p>1$, then $q c_{J}\left(R^{p}\right)$ is unbounded.

The condition in Theorem $\mathrm{A}$ is based on the existence of a geodesic access for the corresponding periodic points. However it is easy to see that periodic points may be non-geodesically accessible even when the Julia set is a Jordan curve. We 
study the existence of independent geodesic accesses for the maps in $\mathbf{W}_{2}$ and $\mathbf{W}_{3}$ (see Proposition 3.1 and Theorem 4.1 below). This leads us to the following results.

Let $L(A)$ be the length (period) of a periodic set $A$ determined by a rational $\operatorname{map} R$.

Theorem B. Let $R \in \mathbf{W}_{3}$ be a rational map of degree $d$ and let $D_{1}$ and $D_{2}$ be attracting or superattracting periodic domains of $R$. Let $d_{i}^{j}$ be the degrees of the restrictions $\left.R\right|_{D_{j}^{i}}$, where $D_{j}^{i}=R^{i}\left(D_{j}\right), j=1,2$. Assume that $L(x), L(y) \leq$ $\min \left(L\left(D_{1}\right), L\left(D_{2}\right)\right)$ and

$$
\frac{d_{1}^{j} d_{2}^{j} \ldots d_{L\left(D_{j}\right)-1}^{j}-1}{2} \leq \sum_{i=1}^{L\left(D_{j}\right)}\left(d_{i}^{j}-1\right), \text { for } j=1,2 .
$$

Then $q c_{J}(R)$ is unbounded.

Corollary B. Let $R \in \mathbf{W}_{3}$ be a rational map of degree $d$ and let the domains $D_{1}$ and $D_{2}$ be attracting (superattracting) and invariant. Assume that $x$ and $y$ are fixed points. Then $q c_{J}(R)$ is unbounded.

Theorem C. Let $R \in \mathbf{W}_{2}$. Let $D$ be the domain and $x$ be the point from the definition of $\mathbf{W}_{2}$. Assume that

(1) the domain $D$ is attracting (superattracting) and $L(x) \leq L(D)$,

(2) Let $d_{i}$ be the degrees of the restrictions $\left.R\right|_{D_{i}}$, where $D_{i}=R^{i}(D)$. Suppose $d_{i}=1$ for all $i$ except $i_{0}$.

Then $q c_{J}(R)$ is unbounded.

Corollary C. Let $R$ be a rational map of degree $d$ with a completely invariant domain D. Assume that the fixed points of $R$ are non-indifferent. Then $q c_{J}(R)$ is unbounded.

Outline of proof. The main tool to prove the above theorems is application of the pinching construction to the Riemann surfaces associated with rational maps.

Let $S_{R}$ be the Riemann surface associated with a rational map $R$. Let $S$ be a component of $S_{R}$ equipped with the canonical hyperbolic metric and let $\gamma \subset S$ be a closed, simple (without self-intersection) geodesic (or a leaf of foliation in the case of foliated Riemann surface, see Remark 1 below). Then there is (see [Str]) a small annular neighborhood $A_{\gamma} \subset S$ of $\gamma$ and a conformal map $h: C \rightarrow A$ from the standard ring $C=\{z: 1 / r<|z|<r\}$ onto $A$ such that $h^{-1}(\gamma)=\{z:|z|=1\}$. Then the modulus $m(A)$ of $A$ is given by $\ln (r) / \pi$.

Consider the quasiconformal map $F(z)=z|z|$. Let $C_{n}=F^{-n}(C)$. Then $C_{n+1} \subset$ $C_{n}$. Denote by $\mu_{n}$ the Beltrami differential of $F^{n}$ on $C_{n}$ and consider on $C$ the new Beltrami differentials $\nu_{n}$ by

$$
\nu_{n}= \begin{cases}0 & \text { on } C \backslash C_{1} ; \\ \mu_{i} & \text { on } C_{i} \backslash C_{i+1}, \text { for } 1 \leq i \leq n-1 ; \\ \mu_{n} & \text { on } C_{n} .\end{cases}
$$

Now transfer $\nu_{n}$ to the full orbit of $D$ by means of $h$ and the covering $P_{R}$. Extend $\nu_{n}$ by zero to the Riemann sphere to obtain a sequence $\omega_{n}$ of Beltrami differentials on $\overline{\mathbb{C}}$ invariant with respect to the map $R$ (that is $\omega_{n}(R(z)) \overline{R^{\prime}(z)}=$ $\left.\omega_{n}(z) R^{\prime}(z)\right)$. The Measurable Riemann Mapping Theorem $([\mathrm{Ab}])$ then provides a sequence $H_{n}$ of quasiconformal automorphisms of the Riemann sphere such that the 
maps $R_{n}=H_{n} \circ R \circ H_{n}^{-1}$ are rational. If for some normalization (for $\left\{H_{n}\right\}$ ) there exists a subsequence $\left\{R_{n_{j}}\right\}$ converging to a map $R_{\infty} \operatorname{such}$ that $\operatorname{deg}\left(R_{\infty}\right)=\operatorname{deg}(R)$, then we call $R_{\infty}$ a pinching deformation of $R$ along the geodesic $\gamma$ and denote this map by $R_{\gamma}$. The sequences $R_{n}$ and $H_{n}$ of rational maps and quasiconformal homeomorphisms we call the pinching sequences of maps and homeomorphisms, respectively.

Roughly speaking, we use Remark 3 below to show that pinching deformations shrink independent accesses to points and that we loose in the limit a portion of the Julia set, contradicting the assumption that $R_{n}$ converges. Thus we add one more line to Sullivan's dictionary between Kleinian groups and Holomorphic dynamics. Later on, Kevin Pilgrim ( $\underline{\text { Pil }})$ pointed out the following analogy:

Independent accesses in Theorem $A$ correspond to cylinders for hyperbolic three manifolds.

From this point of view a reformulation of the question (1) (version (ii)) translates a well-known Thurston compactness result for convex cocompact geometrically finite hyperbolic acylindrical three-manifolds with non-empty incompressible boundary (see $[\mathrm{Thu}]$ ).

\section{Remarks.}

Remark 1. In the foliated cases a geodesic always means a leaf of the foliation. Hence, the pinching deformation is well defined since $F(z)$ commutes with the rotation of the annulus $C$.

Remark 2. Assume that there exists a collection of simple closed geodesics $\gamma_{1}, \ldots, \gamma_{n}$ on $S$ such that $\gamma_{i} \cap \gamma_{j}=\varnothing$ for $i \neq j$. Then the pinching deformations may be defined along these geodesics simultaneously. If the pinching deformation of $R$ along $\gamma_{1}, \ldots, \gamma_{n}$ exists, we denote it by $R_{\gamma_{1}, \ldots, \gamma_{n}}$. (Note: The deformations depend on $A_{1}, A_{2}, \ldots$ )

Remark 3. Let $A_{n}$ be $h^{-1}\left(C_{n}\right)$ and suppose $\Psi_{n}: S \rightarrow S_{R_{n}}$ is a quasiconformal homeomorphism with dilatation equal to $\nu_{n}$. Then it is easy to show that moduli of the annuli $B_{n} \cup B_{n}{ }^{\prime}=\Psi_{n}\left(A_{n} \backslash A_{n+1}\right)$ coincide and are equal to $\ln (r) / 2 \pi$ for all $n$. Furthermore the maps $\Psi_{i} \circ \Psi_{n}^{-1}$ are conformal on both components $K_{n}$ and $K_{n}{ }^{\prime}$ of $\Psi_{n}\left(A \backslash A_{n+1}\right)$ for all $i \geq n+1$, and

$$
m\left(K_{n}\right), m\left(K_{n}{ }^{\prime}\right) \geq \sum_{i=0}^{n} m\left(B_{i}\right)=\frac{n \ln (r)}{2 \pi} .
$$

In particular, the maps $\Psi_{i} \circ \Psi_{n}^{-1}$ are conformal on $\left\{\Psi_{n}(S \backslash A)\right\} \cup\left\{K_{n} \cup K_{n}{ }^{\prime}\right\}$ for all $i \geq n+1$.

We conclude the introduction with the following useful lemma whose proof is an application of the quasiconformal surgery of A. Douady and J. Hubbard ([DH] and M. Shishikura ([Sh] $)$.

Lemma 1. Let $R$ be a rational map and let $D \subset \mathbf{F}(R)$ be an attractive (superattractive) or parabolic periodic domain. Denote by $d_{i}$ the degree of the restriction $\left.R\right|_{D_{i}}$, where $D_{i}=R^{i}(D)$. Then there is a rational map $R_{1} \subset q c_{J}(R)$ and a homeomorphism $h: \mathbf{J}(R) \rightarrow \mathbf{J}\left(R_{1}\right)$ conjugating $R$ to $R_{1}$ such that

(1) the domain $D_{1} \subset \mathbf{F}\left(R_{1}\right)$ bounded by $h(\partial D)$ is attracting or parabolic, respectively; 
(2) in the case of the connected Julia set, for any $i$ the domains $D_{1}^{i}=R\left(D_{1}\right)$ contain $d_{i}-1$ simple critical points of $R_{1}$ and their forward orbits are infinite;

(3) there are no critical points in $\bigcup_{i=1}^{k}\left\{R_{1}^{i}\left(D_{1}\right)\right\}$ having intersecting forward orbits.

Contents. In $\$ 1$ we prove Theorem $A$ and Corollary $\mathrm{A}$ In 92 , we prove the claims in Example $\mathrm{A}[3$ and $\$ 4$ prove Theorems $\mathrm{B}$ and $\mathrm{C}$ as well as Corollaries $\mathrm{B}$ and $\mathrm{C}$

Remark 4. This paper is based on the preprint of 1993, Mak1.

\section{Proofs of Theorem and Corollary}

We start with the next simple lemma.

Lemma 1.1. Let $R$ be a rational map and $\gamma_{1}, \ldots, \gamma_{n} \in S_{R}$ be a collection of simple closed geodesics (or leafs of invariant foliations) such that $\gamma_{i} \cap \gamma_{j}=\varnothing$ for $i \neq j$. Assume that there is no pinching deformation along $\gamma_{1}, \ldots, \gamma_{n}$. Then $q c_{J}(R)$ is unbounded.

Proof. Choose some normalization for the pinching sequence $\left\{H_{n}\right\}$ of quasiconformal homeomorphisms and assume that $q c_{J}(R)$ has compact closure in $R_{a t}$. Then there exists a sequence $\left\{g_{i}\right\}$ of Möbius maps and a subsequence $\left\{H_{n_{i}}\right\}$ such that the maps $R_{i}=g_{i} \circ H_{n_{i}} \circ R \circ H_{n_{i}}^{-1} \circ g_{i}^{-1}$ converge to a rational map $R_{\infty}$ of degree $d=\operatorname{deg}(R)$. The critical and fixed points of $R_{n_{j}}$ converge respectively to critical and fixed points of $R_{\infty}$. Now, let $c_{1}, c_{2}, c_{3}$ be different critical points of $R_{\infty}$ (or critical points and critical values if $R_{\infty}$ does not have 3 different critical points, or critical points and fixed point if $R_{\infty}$ is conjugated with $z \rightarrow z^{d}$ or $z \rightarrow \frac{1}{z^{d}}$ ) and let $x_{1}, x_{2}, x_{3}$ be corresponding points for $R$ such that $g_{i} \circ H_{n_{i}}\left(x_{j}\right) \rightarrow c_{j}$, respectively. Now choose a new normalization for $H_{n}$, namely: $H_{n}=\alpha_{n} \circ \Psi_{n}$, where $\Psi_{n}\left(x_{j}\right)=x_{j}$. Then $g_{i} \circ \alpha_{n_{i}}\left(x_{j}\right) \rightarrow c_{j}$ and hence $\lim \left(g_{i} \circ \alpha_{n_{i}}\right)=g_{0}$ does exist and $g_{0}$ is a non-degenerate Möbius map. We conclude that $\Psi_{n_{i}} \circ R \circ \Psi_{n_{i}}^{-1} \rightarrow g_{0}^{-1} \circ R_{\infty} \circ g_{0}$, contradicting the assumption that there is no pinching deformation.

Next, we divide the proof of Theorem $\mathrm{A}$ into 3 cases, according to whether $R$ belongs to $\mathbf{W}_{1}, \mathbf{W}_{2}$, or $\mathbf{W}_{3}$.

1) $R \in \mathbf{W}_{1}$. To start with we assume that $\mathbf{F}(R)$ contains a cycle of Herman rings of period $k$. Let $D$ be a component of this cycle and $l \subset D$ be a leaf of the invariant foliation which does not intersects orbits of the critical points of $R$. Let $A \subset D$ be a small invariant ring neighborhood of $l$ and $R_{n}$ and $h_{n}$ be the pinching sequences of rational maps and quasiconformal homeomorphisms, respectively. Assume that for some normalization there is a limit map $R_{\infty}$ of the same degree as $R$. Then the maps $h_{n}$ on $C_{1} \cup C_{2}=D \backslash A$ form normal families of conformal maps. Indeed, as in Lemma 1.1 above, consider triples of points $c_{1}, c_{2}, c_{3}$ and $x_{1}, x_{2}, x_{3}$ for maps $R$ and $R_{\infty}$, respectively with $h_{n}\left(c_{i}\right) \rightarrow x_{i}$ (arguments are up to a subsequence). Then the functions from the following family

$$
f_{n}=\frac{h_{n}(z)-h_{n}\left(c_{1}\right)}{h_{n}(z)-h_{n}\left(c_{2}\right)} \frac{h_{n}\left(c_{3}\right)-h_{n}\left(c_{2}\right)}{h_{n}\left(c_{3}\right)-h_{n}\left(c_{1}\right)}
$$

omit the values 0,1 and $\infty$ on $\overline{\mathbb{C}}-\left\{\bigcup_{i} c_{i}\right\}$.

We show that in one of the families $\mathfrak{F}_{i}=\left\{\left.h_{n}\right|_{C_{i}}\right\}, i=1,2$, all limits are constants. Indeed, assume, say $\mathfrak{F}_{1}$, has a non-constant limit map $H_{\infty}$ and $\left\{H_{n_{j}}\right\} \rightarrow H_{\infty}$ is a 
convergent sequence. Then

$$
R_{\infty}^{k}\left(H_{\infty}\left(C_{1}\right)\right)=H_{\infty}\left(C_{1}\right)
$$

and $H_{\infty}\left(C_{1}\right)$ is an annulus with definite modulus. But the moduli $m\left(H_{n_{j}}(A)\right) \rightarrow \infty$ and thus the spherical diameter of $H_{n_{j}}\left(C_{2}\right)$ tends to zero. Hence all limit maps for $\mathfrak{F}_{2}$ are constants. We need the following lemma.

Lemma 1.2. Let $R$ be a rational map of degree $d$. Let $\mathbf{P e r}_{k, n}(R)$ be the set of periodic points of the period $l$ with $k \leq l \leq n$. Then there is an integer $N_{R}$ such that

(1) the set $\operatorname{Per}_{k, n}(R)$ consists of repelling periodic points for any $n \geq k \geq N_{R}$;

(2) for any $N_{1} \geq N \geq N_{R}$, there is a neighborhood $U_{N, N_{1}} \subset \mathbb{C} P^{2 d+1}$ of $R$ such that

$$
\operatorname{card}\left(\mathbf{P e r}_{k, n}(F)\right)=\operatorname{card}\left(\mathbf{P e r}_{k, n}(R)\right)
$$

for all $N \leq k \leq n \leq N_{1}$ and $F \in U_{N, N_{1}}$

(3) for any given $N_{1} \geq N \geq N_{R}$ as well $F_{i} \in U_{N, N_{1}}$ and $\left\{F_{i}\right\} \rightarrow R$, then

$$
\operatorname{Per}_{k, n}\left(F_{i}\right) \rightarrow \operatorname{Per}_{k, n}(R)
$$

uniformly. Moreover for all sufficiently large $i$ there are homeomorphisms $f_{i}$ mapping the sets $\mathbf{P e r}_{k, n}\left(F_{i}\right)$ onto $\mathbf{P e r}_{k, n}(R)$ and conjugating the action $R$ with the action $F_{i}$. The homeomorphisms $f_{i}$ depend continuously on $F_{i}$ and $f_{i} \rightarrow \mathrm{id}$ as $i \rightarrow \infty$.

Proof. The number of non-repelling points of $R$ is bounded and this gives (1). The cases (2) and (3) follow by analyzing the solutions of the equation

$$
F_{t}^{m}(z)-z=0, \text { for } N \leq k \leq m \leq n \leq N_{1},
$$

where $F_{t}(z) \in U_{N, N_{1}}$ and $U_{N, N_{1}}$ is a neighborhood such that all solutions of the equation above with the initial conditions

$$
F_{0}^{m}\left(z_{0}\right)=R^{m}\left(z_{0}\right)=z_{0}, \text { for } z_{0} \in \mathbf{P e r}_{k, n}(R)
$$

are well defined on $U_{N, N_{1}}$.

Now we return back to Theorem A. We have that all limit functions of one of the families $\mathfrak{F}_{i}$ are constants. Then the spherical diameter of one of the components $B_{1}^{j}$ and $B_{2}^{j}$ of $h_{n_{j}}(\overline{\mathbb{C}} \backslash A)$ tends to 0 as $n_{j} \rightarrow \infty$ for any convergent subsequence $\left\{h_{n_{j}}\right\} \subset$ $\left\{h_{n}\right\}$. Let $\operatorname{diam}\left(B_{1}^{j}\right) \rightarrow 0$ and let $x$ and $y$ be different points of $\operatorname{Per}_{k, n}(R) \cap B_{1}$ with $k \geq N_{R_{\infty}}$. Then by the above construction we have $x_{j}=h_{n_{j}}(x)$ and $y_{j}=h_{n_{j}}(y) \in$ $\operatorname{Per}_{k, n}\left(R_{n_{j}}\right) \cap B_{1}^{j}$. Therefore $\lim _{i \rightarrow \infty} x_{j_{i}}=\lim _{i \rightarrow \infty} y_{j_{i}}$ for some $\left\{j_{i}\right\} \subset\{j\}$. This contradicts Lemma 1.2 .

Now we examine the general case. Let $D$ be a non-simply connected Fatou component and $O$ be the periodic component of the forward orbit of $D$. Then $O$ is either attractive (superattractive), parabolic, or Siegel. Let $O=R^{k}(D)$.

Assume first that there is a Jordan curve $\gamma \subset O$ (in the Siegel case $\gamma$ is a leaf of foliation) satisfying:

(1) there exists a small ring neighborhood $\mathcal{A} \subset D$ of $\gamma$ such that $\left.R^{n}\right|_{\mathcal{A}}$ is univalent for all $n \geq 1$;

(2) there is a component $\Omega \subset \bigcup_{n} R^{-n}(\mathcal{A})$ separating components of $\partial D$;

(3) $R^{n}(\mathcal{A}) \cap R^{m}(\mathcal{A})=\varnothing$ for any $n \neq m$ in the parabolic and attractive cases. 
Claim. The space $q c(R)$ is unbounded.

Proof of the claim. Conditions (1), (2), and (3) imply that the projection $P_{R}(\gamma) \subset$ $S_{R}$ is a closed Jordan curve. We may assume $\gamma$ is a geodesic. Under assumption (2) there exists a component $O_{1} \subset D$ of $P_{R}^{-1}\left(S_{R} \backslash P_{R}(\mathcal{A})\right)$ separating components of $\partial D$. Let $h_{n}$ and $R_{n}$ be the pinching sequences of quasiconformal homeomorphisms and rational maps, determined by $\gamma$. Suppose there is a limit map $R_{\infty}$ of degree $d=$ $\operatorname{deg}(R)$. Then there is a subsequence $\left\{n_{j}\right\} \subset\{n\}$ such that the family $\mathfrak{F}=\left\{h_{n_{j}} \mid O_{1}\right\}$ is normal. Lemma 1.2 implies that all limit maps of $\mathfrak{F}$ cannot be constants. Let $H_{\infty}$ be a limit map and $Q$ a component of $\mathbf{F}\left(R_{\infty}\right)$ containing the set $Q_{1}=H_{\infty}\left(O_{1}\right)$. Then $Q_{1}$ separates components of $\partial Q$.

Remark 3 implies that for any $N$ there exists $N_{1}$ such that for any fixed $s \geq N_{1}$ the maps

$$
\psi_{j}=h_{i_{j}} \circ h_{s}^{-1}
$$

are conformal on the component $O_{s} \subset \mathbf{F}\left(R_{s}\right)$ containing the set $h_{s}\left(O_{1}\right)$ and the set $O_{s} \backslash h_{s}\left(O_{1}\right)$ consists of the rings $B_{l}, l=1, \ldots, k, k \geq 2$ with moduli $m\left(B_{s}\right) \geq N$. Therefore, if $\Psi_{\infty}$ is a limit map for $\psi_{j}$, then

$$
\left.\Psi_{\infty}\right|_{h_{s}\left(O_{1}\right)}=\left.H_{\infty} \circ h_{s}^{-1}\right|_{h_{s}\left(O_{1}\right)} .
$$

Moreover any component of $\overline{\mathbb{C}} \backslash H_{\infty}\left(O_{1}\right)$ contains a ring $C_{l}=\Psi_{\infty}\left(B_{l}\right)$ for some $l$ and $\partial C_{l} \cap H_{\infty}\left(\overline{O_{1}}\right) \subset \partial H_{\infty}\left(O_{1}\right)$. We conclude that the set $\overline{\mathbb{C}} \backslash Q$ consists of a finite number of points since the moduli of $C_{l}$ may be arbitrarily large. This contradicts Lemma 1.2

Now we show an existence of a rational map $R_{1} \subset q c_{J}(R)$ and a curve $\gamma \subset \mathbf{F}\left(R_{1}\right)$ satisfying conditions (1), (2), and (3).

Let $O$ be a Siegel disk. Define $\gamma$ to be an invariant leaf whose interior (up to a Möbius change of coordinates) contains all first hits of forward orbits of the critical points of the full orbit of $O$. Note that in our case the full orbit of $O$ contains at least two critical points. Then passing to the branched covering $R^{k}: D \rightarrow O$ it is easy to see that $\gamma$ satisfies conditions (1), (2), and (3).

Let $O$ be an attractive periodic domain of the period $k$. Then we define $\gamma$ to be the boundary of a small disk neighborhood $U$ of an attractive periodic point such that $R(U) \subset U$ and $\left.R\right|_{U}$ is univalent. Then (1) and (3) are satisfied. We now show (2). If $O$ is not simply connected, then there is an iterated preimage of $\gamma$ separating components of $\partial O$ and (2) is satisfied.

If $O$ is simply connected, then all components of $R^{-n}(U) \cap D$ are simply connected for any $n$. Let $U_{n}$ be a component of $R^{-n}(U) \cap D$ containing all first hits of the forward orbits of critical points from the full orbit of $O$. Consider the branched covering $R^{m}: D \rightarrow O$. Then $\partial U_{n}$ is homotopic to $\partial O$, thus $R^{-l}\left(\partial U_{n}\right)$ is homotopic to $\partial D$ and $(2)$ is satisfied.

The superattractive case is reduced to the attractive one by Lemma 1

Let $O$ be a parabolic periodic domain. Then by Lemma 1 we have a map $R_{1} \in q c_{J}(R)$ and a homeomorphism $h: \mathbf{J}(R) \rightarrow \mathbf{J}\left(R_{1}\right)$ such that

(1) the component $O_{1}$ bounded by $h(\partial O)$ is parabolic, and

(2) the sets of forward orbits of critical values belonging to the forward orbit of $O_{1}$ are mutually disjoint.

Then by Mak, Theorem 1] there is a horoneighborhood $U \subset O_{1}$ of the parabolic point such that 
(1) $\left.R\right|_{U}$ is univalent, $R(U) \subset U$ and

(2) $U \backslash R(U)$ contains all first hits of the orbits of critical values from the full orbit of $O_{1}$.

Let $\gamma \subset U \backslash R(U)$ be a closed Jordan curve whose interior contains all first hits from (2). Again consider the branched covering $R^{l}: D_{1} \rightarrow O_{1}$, where $D_{1}$ is bounded by $h(\partial D)$. Then we conclude that $\gamma$ satisfies (1), (2), and (3) since the interior of $\gamma$ contains all critical values of this covering and $\pi_{1}\left(D_{1}\right) \neq 1$.

The case $R \in \mathbf{W}_{1}$ is proved.

2) $R \in \mathbf{W}_{2}$. We start with Maskit inequalities.

Lemma 1.3 (The Maskit inequalities). Let $\mathbf{S}$ be a hyperbolic surface of finite topological type (that is $\pi_{1}(\mathbf{S})$ is finitely generated) and $\gamma$ be a simple closed geodesic on $\mathbf{S}$. If $\mathcal{A}$ is a ring neighborhood of $\gamma$ in $\mathbf{S}$, let $m(\mathcal{A})$ be the extremal length of the family of all rectifiable curves in $\mathcal{A}$ freely homotopic to $\gamma$. Then

1) $L_{\gamma} \leq 2 \pi m(\mathcal{A}) \leq \pi L_{\gamma} \exp \left(L_{\gamma} / 2\right)$, where $L_{\gamma}$ is the length of $\gamma$.

2) Let $\mathbf{T}$ be a torus and $p: \mathbb{C} \backslash\{0\} \rightarrow \mathbf{T}$ the unbranched covering with the covering group $G=\langle g=\lambda z\rangle$, for some $|\lambda|>1$. Let $[c]$ be a generator of $\pi_{1}(\mathbb{C} \backslash\{0\})$ represented by a curve $c$ with counterclockwise orientation. Let $\Sigma, \Gamma$ be the generators of the fundamental group of the torus $\mathbf{T}$ such that

$$
p^{*}(g)=\Gamma, \quad p^{*}([c])=\Sigma .
$$

Let $\gamma$ be a simple closed Jordan curve homologous to a loop $\Sigma^{-p} \Gamma^{q}$. Then the lifting $p^{-1}(\gamma)$ consists of $q$ curves $\gamma_{1}, \ldots, \gamma_{q}$. If $\mathcal{A} \subset \mathbf{T}$ is a small ring neighborhood of $\gamma$, then

$$
\frac{\ln ^{2}|\lambda|+(\arg (\lambda)-2 \pi p / q)^{2}}{2 \pi \ln |\lambda|} \leq m(\mathcal{A}) .
$$

The number $p / q$ is called the combinatorial rotation number of the curves $\gamma_{1}, \ldots, \gamma_{q}$ with respect to the point $z=0$.

If $|\lambda|<1$, then in the above terms we have that

$$
\frac{\ln ^{2}|\lambda|+(\arg (\lambda)+2 \pi p / q)^{2}}{2 \pi \ln |\lambda|} \leq m(\mathcal{A}),
$$

and the combinatorial rotation number of curves $\gamma_{1}, \ldots, \gamma_{q}$ with respect to the point $z=0$ is equal to $-p / q$. In other words, the combinatorial rotation numbers $\alpha_{0}, \alpha_{\infty}$ of the curves $\gamma_{1}, \ldots, \gamma_{q}$ with respect to 0 and $\infty$, respectively, satisfy the equation

$$
\alpha_{0}+\alpha_{\infty}=0 .
$$

3) Let $B$ be a topological ring in $\mathbb{C} \backslash\{0\}$ separating 0 and $\infty$ and suppose $g^{n}(B) \cap$ $B=\varnothing$ for some $g(z)=\lambda z,|\lambda|>1$, and any $n$. If $B$ is conformally equivalent to $\mathcal{A}$, then

$$
\frac{2 \pi}{\ln |\lambda|} \leq m(\mathcal{A}) .
$$

Moreover all above inequalities are quasiconformally quasinvariant.

Proof. See [Mas1], Mas2]. The case (1) is the corollary of the well known collar lemma. The cases (2) and (3) are results of comparison of $m(B)$ with the Euclidean metric on $\mathcal{A}$. 
Proposition 1.1. Let $R$ be a rational map and $D$ be an attractive (parabolic) periodic domain. Let $S_{D} \subset S_{R}$ be the Riemann surface associated with $D$ and $\gamma_{1}, \ldots, \gamma_{n}$ closed simple geodesics on $S_{D}$ with non-empty sets of main components and $\gamma_{i} \cap \gamma_{j}=\varnothing$ for $i \neq j$. Assume the pinching deformation $R_{\gamma_{1}, \ldots, \gamma_{n}}$ does exist. Then there is a continuous map $h: D \rightarrow \overline{\mathbb{C}}$ such that $h$ is a diffeomorphism on $D \backslash P^{-1}\left(\gamma_{1}, \ldots, \gamma_{n}\right)$ and

$$
h \circ R=R_{\gamma_{1}, \ldots, \gamma_{n}} \circ h .
$$

Proof. Without loss of generality we can analyze the case with only one geodesic $\gamma \subset S_{D}$.

Choose some ring neighborhood $\mathcal{A} \subset \mathbf{S}_{D}$ of $\gamma$. Let $D_{j}, j=1, \ldots, m$ be the components of $D \backslash P_{R}^{-1}(\mathcal{A})$ touching the attractive (parabolic) point $z_{0} \in D\left(z_{0} \in \partial D\right)$. Then there is an integer $k$ such that for any $l \geq 1, R^{k l}\left(D_{j}\right)=D_{j}$ and each $D_{j}$ contains at least one critical point of $R^{k l}$. Consider pinching sequences of quasiconformal maps $h_{i}$. Then there is a subsequence $\left\{i_{l}\right\} \subset\{i\}$ such that families $\mathfrak{F}_{j}=\left\{\left.h_{i_{l}}\right|_{D_{j}}\right\}$ are normal. Note that we can assume that the sequence $\left\{\left.h_{i_{l}}\right|_{D_{j}}\right\}$ is convergent for any $j$. If $R_{l}=h_{i_{l}} \circ R \circ h_{i_{l}}^{-1}$ and $z_{l}=h_{i_{l}}\left(z_{0}\right)$, then the limit $x=\lim z_{l}$ is a parabolic periodic point. To see this, if $z_{l}$ are parabolic periodic points, then $x$ is parabolic obviously. Now assume that $z_{l}$ are attractive points. Consider the geodesic $\gamma$. Since $\gamma$ has main components, $\gamma$ is homotopic to a loop $\Sigma^{-p} \Gamma^{q}$ (see the Maskit inequalities (2)) with non-zero $q$. Then by Remark 3 and the Maskit inequalities (2) we see the multiplier of the point $x$ is equal to $\exp \left(-2 \pi i \frac{p}{q}\right)$.

Denote by $H_{j}$ the limit maps for $\left\{\left.h_{i_{l}}\right|_{D_{j}}\right\}$, respectively. Then for all $j$ these maps satisfy:

(i) $H_{j} \neq$ const,

(ii) if $R^{k}\left(D_{i}\right)=D_{j}$, then

$$
H_{j} \circ R^{k}=R_{\gamma}^{k} \circ H_{i} .
$$

To prove (i), suppose $H_{j}=a$ for some $j$ and number $a \in \mathbb{C}$. The region $D_{j}$ contains a critical point of $R_{\gamma}^{n}$, thus $a$ has to be a critical point for $R_{\gamma}^{n}$. The point $x$ must coincide with $a$. Then $a$ is a periodic point for $R_{\gamma}^{n}$, contradicting the parabolicity of $x$.

(ii) is obvious.

Denote by $O_{j}$ the component of $\mathbf{F}\left(R_{\gamma}\right)$ containing $H_{j}\left(D_{j}\right)$. Then all these components touch the point $x=H_{1}\left(z_{0}\right)=\cdots=H_{m}\left(z_{0}\right)$ and $O_{j}$ belongs to the immediate basin of attraction of $x$ for each $j=1, \ldots, m$.

Claim. The set $O_{i} \backslash H_{i}\left(D_{i}\right)$ consists of the full orbit of two, invariant under $R_{\gamma}^{n=l k}$, topological disks $w_{1}^{i}$ and $w_{2}^{i}$ both touching the point $x$. These disks are called petals of the point $x$ in $O_{i}$.

Proof of Claim. To start with we show that $O_{i} \backslash H_{i}\left(D_{i}\right)$ does not contain critical points. Otherwise $H_{i}$ induces a conformal map $H_{i}^{*}$ from the component $B \subset S_{D} \backslash \mathcal{A}$ into $S_{O_{i}}$ (or from components $B_{1}$ and $B_{2} \subset \mathbf{S}_{D} \backslash \mathcal{A}$ in the parabolic case) such that there is a component of $S_{O_{i}} \backslash H_{i}^{*}(B)$ containing a marked point $\alpha$. By definition of the pinching deformation we know that the maps $\varphi_{j}=h_{j} \circ h_{s}^{-1}$ are induced by the maps $\Phi_{j}=\Psi_{j} \circ \Psi_{s}^{-1}$ (see Remark 3) which are conformal on the rings 
$K_{s} \cup K_{s}^{\prime} \subset \Psi_{s}(\mathcal{A})$. The moduli of these rings satisfy

$$
m\left(K_{s}\right), m\left(K_{s}^{\prime}\right) \geq s \frac{\ln r}{2 \pi} .
$$

Therefore the map $H_{i}^{*} \circ h_{s}^{-1}$ may be extended to a conformal embedding $\hat{H}_{i}$ from $K_{s} \cup K_{s}^{\prime}$ into $S_{O_{i}}$. Thus for all large $s$ the set $\hat{H}_{i}\left(K_{s} \cup K_{s}^{\prime}\right)$ contains the point $\alpha$. This contradicts the number of marked points of the component $S_{D} \backslash \mathcal{A}$.

Hence the set $S_{O_{i}} \backslash H_{i}(B)$ consists of two punctured disks $W_{1}^{i}$ and $W_{2}^{i}$ without marked points. Therefore we conclude that the set $W_{i}=P_{R_{\gamma}}^{-1}\left(W_{1}^{i} \cup W_{2}^{i}\right)$ is the full orbit of two components $w_{1}^{i}$ and $w_{2}^{i} \subset W_{i} \cap O_{i}$ touching the point $x$. The map $R_{\gamma}^{n}$ restricted to any component of $W_{i}$ is one-to-one.

This Claim implies that the map $H_{i}$ defines a continuous map on any component $\beta$ of $\partial D_{i} \cap P_{R}^{-1}(\partial \mathcal{A})$ and $H_{i}\left(x_{\beta}\right)=H_{i}\left(y_{\beta}\right)$, where $x_{\beta} \cup y_{\beta}=\partial \beta$. On $\beta \backslash \partial \beta$ the map $H_{i}$ is a homeomorphism.

Denote by $B^{*}$ the set $S_{D} \backslash \gamma$. Then $B^{*} \supset B\left(\supset\left\{B_{1} \cup B_{2}\right\}\right.$ in the parabolic case). Let $\Psi_{i}^{*}$ be a diffeomorphism from $B^{*}$ on $S_{O_{i}}$ coinciding with $H^{*}$ on $B$. This diffeomorphism is unique up to isotopy on $B^{*}$.

Note that actually $\Psi_{i}^{*}$ is a limit of $\left\{h_{i_{l}}\right\}$ since, by construction, the homeomorphisms $h_{i}$ have locally uniformly bounded distortion on any compact subset in $B^{*}$.

Then $\Psi_{i}^{*}$ may be lifted from the component $D_{i}^{*}$ of $D \backslash P_{R}^{-1}(\gamma)$ containing the set $D_{i}$ to a diffeomorphism $\Psi_{i}$ from $D_{i}^{*}$ onto $O_{i}$ conjugating the action $R^{n}$ with the action $R_{\gamma}^{n}$. Let $C$ be a component of $D_{i}^{*} \backslash D_{i}$ and $\beta=\partial C \cap P_{R}^{-1}(\gamma)$, then $\Psi_{i}(C \cup \beta)=$ $\bar{w}$ for some $w \in W_{i}$. Define $\Psi_{i}$ on $\beta$ as $\Psi_{i}(\partial \beta)$. Then it is easy to see that by means of the actions of $R^{n}$ and $R_{\gamma}^{n}$ we obtain a continuous map from $D_{i}^{*} \cup\left\{\overline{D_{i}^{*}} \cap P_{R}^{-1}(\gamma)\right\}$ onto $O_{i}$. If $\beta \in \overline{D_{i}^{*}} \cup \overline{D_{j}^{*}} \cap P_{R}^{-1}(\gamma)$, then $\Psi_{i}(\beta)=\Psi_{j}(\beta)$ by construction. Therefore the maps $\Psi_{i}$ define a continuous map $\Psi$ from $\bigcup_{i}\left\{D_{i}^{*} \cup\left\{\overline{D_{i}^{*}} \cap P_{R}^{-1}(\gamma)\right\}\right\}$ onto $\bigcup_{i} O_{i}$, which semi-conjugates the action $R^{k}$ with the action $R_{\gamma}^{k}$. By induction we complete this proposition.

Theorem 1.1. Let $R$ be a rational map with connected Julia set and no superattractive periodic domains. Let $\gamma_{1}, \ldots, \gamma_{n} \subset S_{R}$ be a family of closed simple geodesics with $\gamma_{i} \cap \gamma_{j}=\varnothing$ if $i \neq j$. Assume that the pinching deformation $R_{\gamma_{1}, \ldots, \gamma_{n}}$ does exist. Then there is a continuous map $h$ from $\mathbf{F}(R)$ into $\overline{\mathbb{C}}$ such that $h$ is a diffeomorphism on $\mathbf{F}(R) \backslash\left\{\bigcup_{i} P_{R}^{-1}\left(\gamma_{i}\right)\right\}$ and

$$
h \circ R=R_{\gamma_{1}, \ldots, \gamma_{n}} \circ h .
$$

Moreover $h$ maps each connected component of $\bigcup_{i} P_{R}^{-1}\left(\gamma_{i}\right)$ to a single point.

Proof. Let $\left\{R_{n_{i}}\right\}$ be a convergent pinching subsequence of rational maps. Then,

(1) critical points of $R_{n_{i}}$ converge uniformly to critical points of $R_{\gamma_{1}, \ldots, \gamma_{n}}$,

(2) non-repelling periodic points of $R_{n_{i}}$ converge uniformly to non-repelling periodic points of $R_{\gamma_{1}, \ldots, \gamma_{n}}$, and

(3) the repelling periodic points of large enough period converge to the repelling periodic points of $R_{\gamma_{1}, \ldots, \gamma_{n}}$ by Lemma 1.2.

Thus we conclude that there exists a subsequence $\left\{h_{i}\right\} \subset\left\{h_{n_{i}}\right\}$ converging on $\mathbf{F}(R) \backslash \bigcup_{i} P_{R}^{-1}\left(\mathcal{A}_{i}\right)$ to a non-constant conformal map, where $\mathcal{A}_{i} \subset S_{R}$ are some annular neighborhoods of $\gamma_{i}$, respectively. The argument given in Proposition 1.1 completes the proof. 
Theorem 1.1 implies that if a pinching deformation $R_{\gamma}$ does exist along a geodesic $\gamma$, then the set $P_{R}^{-1}(\gamma)$ does not contain finitely many components whose closure separates the Riemann sphere. This is an important observation for the remainder of the proofs.

Let $R \in \mathbf{W}_{2}$ and $R_{1} \in q c_{J}(R)$. Let $D$ be a component of $\mathbf{F}\left(R_{1}\right)$ and $x \in \partial D$ be an accessible periodic point with at least two independent accesses.

Under the assumptions we have, there is either one geodesic $\gamma$ and two main components $\beta_{1}$ and $\beta_{2}$ of the lifting of $\gamma$ defining the different accesses to $x$, or there are two geodesics $\gamma_{1}$ and $\gamma_{2}$ with main components $\beta_{1}$ and $\beta_{2}$ defining two different accesses to $x$, respectively. Again, consider pinching along either $\gamma$ or $\gamma_{1} \cup \gamma_{2}$. If either $R_{\gamma}$ or $R_{\gamma_{1}, \gamma_{2}}$ does exist, then Theorem 1.1 gives a continuous map $h$ from $D$ into $\overline{\mathbb{C}}$ semi-conjugating $R$ with $R_{\gamma}$. The restriction of $h$ on the closure of any component of $P_{R}^{-1}(\gamma)$ is a constant map. In both cases the set $\overline{\beta_{1}} \cup \overline{\beta_{2}}$ defines a closed Jordan curve separating $\overline{\mathbb{C}}$ such that both components $B_{1}$ and $B_{2}$ of $\overline{\mathbb{C}} \backslash\left\{\overline{\beta_{1}} \cup \overline{\beta_{2}}\right\}$ contain points of $\mathbf{J}\left(R_{1}\right)$. Hence we have

$$
\left.h\right|_{\left\{\overline{\beta_{1}} \cap \overline{\beta_{2}}\right\}}=\text { const }
$$

and we loose a portion of the Julia set by taking a limit. This contradicts Lemma 1.2, and proves the case when $R \in \mathbf{W}_{2}$.

3) $R \in \mathbf{W}_{3}$. Consider $R_{1}$ and the geodesics generating accesses from $D_{1}$ and $D_{2}$ to periodic points $x$ and $y$, respectively. Again pinching along these geodesics gives the desired contradiction. The case $R \in \mathbf{W}_{3}$ is done.

Proof of Corollary A. To start with we consider the Blaschke maps with connected Julia sets.

Proposition 1.2. Let $B$ be a Blaschke map of degree $d>2$ with connected Julia set, and $\mathbf{F i x}(B)$ be the set of fixed repelling points of $B$. Let the component $S_{\Delta} \subset S_{B}$ associated with unit disk $\Delta$ be either a torus with $d-1$ punctured points or a sphere with $d+1$ punctured points. Then for any point $x \in \mathbf{F i x}(B)$ there exists a geodesic $\gamma_{x} \subset S_{\Delta}$ such that the main component $\beta_{x}$ of the lift of $\gamma_{x}$ lands at the point $x$. Moreover $\beta_{x} \cap \beta_{y}=\varnothing$ for $x \neq y \in \mathbf{F i x}(B)$.

Proof. Let $S$ and $T$ be natural generators of $\pi_{1}\left(\mathbf{T}_{\Delta}\right)$ (in the parabolic case $T$ is the natural generator of $\left.\pi_{1}\left(\mathbf{S p}_{\Delta}\right)\right)$ such that $P_{B}^{*}(B)=T$.

Let $\gamma_{1}, \ldots, \gamma_{d-1}$ be different geodesics on $S_{\Delta}$ freely homotopic to $T$ on $\mathbf{T}_{\Delta}$ such that $S_{\Delta} \backslash\left\{\bigcup_{i} \gamma_{i}\right\}$ consists of the union of non-degenerate rings with only one puncture. In the parabolic case let $\gamma_{1}, \ldots, \gamma_{d-2}$ be different geodesics on $S_{\Delta}$ freely homotopic to $T$ on $\mathbf{S p}_{\Delta}$ such that $S_{\Delta} \backslash\left\{\bigcup_{i} \gamma_{i}\right\}$ consists of two twice punctured disks (each disk containing exactly one puncture of $\mathbf{S} \mathbf{p}_{\Delta}$ ) and the union of nondegenerate rings with only one puncture.

Now, let $\beta_{i}$ be the main components of the lifting of $\gamma_{i}$, respectively. Then,

(1) $\beta_{i}$ is the unique main component of $P_{B}^{-1}\left(\gamma_{i}\right)$;

(2) $\beta_{i}$ lands at some point $x_{i} \in \mathbf{F i x}(B)$;

(3) $B\left(\beta_{i}\right)=\beta_{i}$ and $\left.B\right|_{\beta_{i}}$ is one-to-one.

Assume that two $\beta_{i} \neq \beta_{j}$ land at a common point $y$. Then the interior $L \subset \Delta$ of the loop $\overline{\beta_{i}} \cup \overline{\beta_{j}} \subset \bar{\Delta}$ contains at least one critical point of $B$. But $L$ cannot contain components of $B^{-n}\left(\overline{\beta_{i}} \cup \overline{\beta_{j}}\right)$ for any $n \geq 0$, because $L \cap \partial \Delta=y$. The conditions (1)-(3) imply $B(L)=L$ and $\left.B\right|_{L}$ is one-to-one, a contradiction. 
Now let $R \in \mathbf{W}_{2} \cup \mathbf{W}_{3}$ and $N(R)$ be the minimal integer such that for the map $R_{1}=R^{N(R)}$ in the definition of $\mathbf{W}_{2} \cup \mathbf{W}_{3}$ the domains $D, D_{1}, D_{2}$ are invariant, the periodic points $x$ and $y$ are fixed, and all accesses to the points $x$ and $y$ from $D, D_{1}, D_{2}$ are invariant as well.

By Lemma 1 we find a rational map $R_{2} \in q c_{J}\left(R_{1}\right)$ such that the Fatou set of $R_{2}$ does not contain a superattractive periodic domain. If $O$ is a periodic domain of $\mathbf{F}\left(R_{2}\right)$ and $x \in O$ is a critical point, then $R_{2}^{\prime \prime}(x) \neq 0$ and the forward orbit of $x$ does not intersect the forward orbit of any other critical point (or a periodic point, whenever $O$ is an attractive periodic domain). Then in the case $R_{2} \in \mathbf{W}_{2}$ the component $S_{D} \subset S_{R_{2}}$ is either a torus with $d-1$ punctures or a sphere with $d-3$ punctures, where $d=\operatorname{deg}\left\{\left.R_{2}\right|_{D}\right\}$. In the case $\mathbf{R}_{2} \in \mathbf{W}_{3}$ we have a similar conclusion for $S_{D_{1}}$ and $S_{D_{2}}$.

Therefore, we complete the proof of Corollary A by considering the Blaschke models for either the actions $R_{2}: D \rightarrow D$ or the actions $R_{2}: D_{i} \rightarrow D_{i}, i=1,2$, and applying Proposition 1.2 and Theorem $\mathrm{A}$.

\section{Example $\mathrm{A}$}

Let $R$ be a rational map with a completely invariant domain $D \subset \mathbf{F}(R)$ and let $\mathbf{T}\left(S_{R}\right)=\mathbf{T}\left(S_{D}\right) \times \mathbf{T}\left(S_{1}\right) \times \cdots \times \mathbf{T}\left(S_{n}\right)$ be the Teichmüller space of the Riemann surface $S_{R}$. Then (see $\underline{\underline{S}}$ ) there is a branched covering $\pi$ from $\mathbf{T}\left(S_{R}\right)$ into $R_{a} t_{d}$. In the absence of invariant line fields on $\mathbf{J}(R)$ the image $\pi\left(\mathbf{T}\left(S_{R}\right)\right)$ coincides with $q c(R)$.

Let $S_{D} \subset S_{R}$ be the component associated with $D$ and $\mu \in \mathbf{T}\left(S_{D}\right)$ be a conformal structure. Denote by $B_{\mu}$ the set $\left\{\left(\omega, \nu_{1}, \ldots, \nu_{n}\right) \in \mathbf{T}\left(S_{D}\right) \times \mathbf{T}\left(S_{1}\right) \times \cdots \times \mathbf{T}\left(S_{n}\right)\right.$; $\omega \equiv \mu\}$. Then we have the following simple conclusion.

Proposition 2.1. Let $R$ be a rational map with a completely invariant domain $D$ and $\mu \in \mathbf{T}\left(S_{D}\right)$ be a conformal structure. Then the closure of $\pi\left(B_{\mu}\right)$ in Rat R $_{d}$ is a compact subset.

Proof. Let $R_{i}$ be any sequence from $\pi\left(B_{\mu}\right)$ and $h_{i}$ be a sequence of quasiconformal automorphisms of the Riemann sphere such that $R_{i}=h_{i} \circ R_{1} \circ h_{i}^{-1}$, where $h_{1}=i d$. Then we can assume that

(1) the maps $h_{i}$ are conformal on the completely invariant domain $D_{1}$ of $R_{1}$;

(2) the point $\infty$ belongs to $D_{1}$ and $h_{i}(z)=z+O(1 / z)$ for $z \rightarrow \infty$.

In other words the maps $\left\{\left.h_{i}\right|_{D_{1}}\right\}$ define a normal family. Let $R_{\infty}$ be a limit map for a subsequence $\left\{R_{i_{j}}\right\}$. Then $R_{\infty}$ is a rational map. If $H_{\infty}$ is a limit map for $\left\{\left.h_{i_{j}}\right|_{D_{1}}\right\}$, then by $(2) H_{\infty} \neq$ const and $R_{\infty}=H_{\infty} \circ R \circ H_{\infty}^{-1}$ on $H_{\infty}\left(D_{1}\right)$. We conclude that $\operatorname{deg}(R)=\operatorname{deg}\left(R_{\infty}\right)$. The proposition is proved.

Case 1. Let $R_{1}$ and $R_{2} \in q c_{J}\left(R_{1}\right)$ be parabolic degree two maps with connected Julia sets. Let $h_{1}$ and $h_{2}$ be conformal maps from completely invariant domains $D_{1}$ and $D_{2}$ respectively onto the upper half plane mapping the parabolic fixed points onto $\infty$, respectively, and such that $h_{1} \circ R_{1} \circ h_{1}^{-1}=h_{2} \circ R_{2} \circ h_{2}^{-1}=z-\frac{1}{z}$. If $h$ is a quasiconformal map from the definition of $q c_{J}(R)$, then $H=h_{2} \circ h \circ h_{1}^{-1}$ is the identity on the real line (since $H$ preserves the orientation, commutes with $z-\frac{1}{z}$ and fixes the point $\infty$ ). Therefore by setting

$$
\widehat{h}= \begin{cases}h_{2}^{-1} \circ h_{1} & \text { on } D_{1} \\ h & \text { on } U_{h} \backslash D_{1}\end{cases}
$$


we obtain an extension of $h$ to $D_{1}$. The arguments of Proposition 2.1 complete the proof of the case (1).

Case 2. Let $S$ be an invariant union of periodic Fatou components for a given rational map $R$. Suppose that $\partial S$ contains a periodic point $x$ of period $n$. Consider the accesses from interior of $S$ to $x$. An access $(x,[\gamma])$ is periodic if there is an integer $k>1$, such that the access $\left(R^{k}(x),\left[R^{k}(\gamma)\right]\right)$ defines the same access $(x,[\gamma])$. Then following [GM] we say that the periodic point $x$ has a combinatorial rotation number $\frac{p}{q}$ if there is a periodic access $(x,[\gamma])$, such that $\gamma$ performs a $\frac{p}{q}$ rotation around $x$ under iterations of $R^{n}$.

Let $R$ be a parabolic degree two map with connected Julia set. Assume that $R$ has an attractive periodic point $z$ of the period $k \geq 2$. Then $R$ has a repelling fixed point $x$ with non-trivial combinatorial rotation number $\alpha=\frac{p}{k}$. Choose $R_{1}=R^{k}$. Then Proposition 1.2 and Theorem $\mathrm{A}$ imply that the space $q c_{J}\left(R_{1}\right)$ is unbounded in $R_{a t}{ }_{2^{k}}$. This gives (2). This shows that Theorem $\mathrm{B}$ fails in general for maps from $\mathbf{W}_{2}$.

\section{Proofs of Theorem B and Corollary B}

Definition 3.1. Let $D$ be an invariant attractive domain for a given rational map $R$. An annulus $\mathcal{C} \subset D$ is called a fundamental domain if $P_{R}(\mathcal{C})=\mathbf{S}_{D}$ and the restriction of $P_{R}$ to the interior of $\mathcal{C}$ is one-to-one and to $\partial \mathcal{C}$ is two-to-one.

A fundamental domain $\mathcal{C}$ is called tame if the interior of $\mathcal{C}$ contains all critical values of $R$ in $D$ and all first hits of the forward orbits of critical points belonging to the back orbit of $D$ minus the back orbit of the attractive point $z \in D$.

Remark 3.1. Let $D$ be an attractive periodic domain of period $m$ for a given rational map $R$ and suppose there are no critical points in the forward orbit of $D$ whose forward orbits intersect. Then there is a tame fundamental domain $\mathcal{C} \subset D$ for $R^{m}$ (see [Mak, Theorem 1]).

Proposition 3.1. Let $B: \Delta \rightarrow \Delta$ be a hyperbolic Blaschke map of degree $d \geq 2$ with connected Julia set. Assume the number critical values of $B$ in the unit disk $\Delta$ is greater than $\frac{d-1}{2}$. Then any two fixed points $x$ and $y \in \partial \Delta$ are independent accessible from $\Delta$.

Proof. Denote by $\mathbf{F i x}_{B}$ the set of all repelling fixed points of $B$ and fix a tame fundamental domain $\mathcal{C}$. Consider the torus $\mathbf{T}_{\Delta}$. Let $\pi_{1}\left(\mathbf{T}_{\Delta}\right)$ be generated by $\mathrm{S}=\left[P_{B}(\partial \mathcal{C})\right]$ and $T=P_{B}^{*}(B)$. Further, as in Proposition 1.2, let $\gamma_{1}, \ldots, \gamma_{m}$ be different geodesics on $S_{\Delta}$ freely homotopic to $T$ and such that $S_{\Delta} \backslash\left\{\bigcup_{i} \gamma_{i}\right\}$ is a union of non-degenerate annuli with only one puncture. Then the main components $\beta_{i}$ of the geodesics $\gamma_{i}$ land at different repelling fixed points of $B$. Denote by $A$ the set of all these points. Then $\operatorname{card}(A)>\frac{d-1}{2}$.

Now, let $\alpha \subset S_{\Delta} \subset \mathbf{T}_{\Delta}$ be a geodesic freely homotopic to $S$ and let $\mathcal{A} \subset S_{\Delta}$ be a small annular neighborhood of $S$. Consider the twist map $\varphi: \mathcal{A} \rightarrow \mathcal{A}$, given by

$$
\varphi(t, \theta)=(t, \theta+2 \pi(t-1))
$$

in the annular coordinates $\langle t, \theta\rangle, 1 \leq t \leq 2,0 \leq \theta \leq 2 \pi$ on $\mathcal{A}$. Then tameness of the fundamental domain $\mathcal{C}$ implies that

(1) $\varphi$ may be extended to a homeomorphism $\Phi: \bar{\Delta} \rightarrow \bar{\Delta}$ commuting with $B$ and

(2) $\left.\Phi\right|_{\partial \Delta}$ is a map of order $d-1$. In other words $\Phi$ acts transitively on the set of fixed points of $B$. 
Now our goal is to show the existence of the integer $k<d-1$ such that $\Phi^{k}(A)$ contains both points $x$ and $y$.

Without loss of generality we may assume that $x \in A$. Let $n_{1}=0<n_{2}<\cdots<$ $n_{m}<d-1$ be a collection of non-negative integers such that $\Phi^{n_{i}}(x) \neq x$ for $i \neq 1$ and $\Phi^{n_{i}}(x) \in A$. Assume that $y \notin \bigcup_{i=1}^{m} \Phi^{n_{i}}(A)$. Then the points $y_{i}=\Phi^{-n_{i}}(y) \notin A$ for $i=1, \ldots, m$. But $m>\frac{d-1}{2}$ and hence $\operatorname{card}\left(\mathbf{F i x}_{B}\right) \geq \operatorname{card}\left(A \cup\left\{\bigcup_{i=1}^{m} y_{i}\right\}\right)>$ $m+\frac{d-1}{2}>d-1$, a contradiction.

Now, return back to Theorem $\mathbb{B}$. Let $N$ be $\min \left(L\left(D_{1}\right), L\left(D_{2}\right)\right)$. We use Lemma 1 to find a rational map $R_{1} \in q c_{J}(R) \subset \mathbf{W}_{3}$ such that

(1) the respective periodic domains of $\mathbf{F}(R)$ (again denoted by $D_{1}$ and $D_{2}$ ) are attracting;

(2) there are no critical points in forward orbits of $D_{1}$ and $D_{2}$ having common full orbit;

(3) if $\ell_{j}$ is a number of punctures of $S_{D_{j}}$, then

$$
2 \ell_{j}>d_{1}^{j} d_{2}^{j} \ldots d_{L\left(D_{j}\right)-1}^{j}-1
$$

Suppose $S_{D_{1}}=S_{D_{2}}$. Then it is easy to see that all accesses to $x$ and $y$ from $D_{1}$ and $D_{2}$ are $R_{1}^{N}$-invariant. If $h$ is a Riemann map from $D_{1}$ onto the unit disk $\Delta$ and $B=h \circ R_{1}^{N} \circ h^{-1}: \Delta \rightarrow \Delta$ is the Blaschke model for $R_{1}^{N}: D_{1} \rightarrow D_{1}$, then there are two different fixed points $x^{\prime}$ and $y^{\prime}$ on $\partial \Delta$ such that $\lim _{r \rightarrow 1} h\left(r x^{\prime}\right)=x$ and $\lim _{r \rightarrow 1} h\left(r y^{\prime}\right)=y$. We observe that $B$ satisfies the assumptions of Proposition 3.1. Thus Proposition 3.1 and Theorem $\mathrm{A}$ complete the proof of this case.

Suppose $S_{D_{1}}$ and $S_{D_{2}}$ are different. If we show that all accesses from $D_{1}$ and $D_{2}$ to the points $x$ and $y$ are invariant, then by the argument above we complete the proof of Corollary B.

One can show (using a linearization near the point $x$ ) that if one access from either $D_{1}$ or $D_{2}$ is invariant, then all accesses from $D_{1}$ and $D_{2}$ are invariant.

Let $B$ be the component of $\overline{\mathbb{C}} \backslash D_{1}$ containing $\overline{D_{2}}$. Then $R_{1}^{k N}\left(\overline{D_{2}}\right) \subset B$ for all $k$. Therefore the accesses from $D_{1}$ to $x$ and $y$ are invariant. Theorem $\mathbb{B}$ and Corollary B are proved.

\section{Proof of Theorem C and Corollary C}

Let $R: \partial \Delta \rightarrow \partial \Delta$ be a continuous endomorphism. Choose a natural order on $\partial \Delta$, that is $x=\exp (2 i \pi t) \leq y=\exp (2 i \pi \theta)$ if $0 \leq t \leq \theta<1$. Let $X=$ $\left(x_{1}<x_{2}<\cdots<x_{n}\right)$ be a periodic cycle of $R$ of period $n$ and assume there is no $k<n$ such that $R^{k}\left(x_{i}\right)=x_{i}$ for any $i$. Denote by $\langle x, y\rangle$ the arc going from $x$ to $y$ in the counterclockwise direction. A map $f_{X}$ is called a linearization of $R$ with respect to $X$ if $f_{X}\left(x_{i}\right)=R\left(x_{i}\right)$ and the map $f_{X}$ is linear on the arcs $\left\langle x_{i}, x_{i+1}\right\rangle$ in the coordinates $0 \leq \theta \leq 2 \pi$, where $x=\exp (2 i \pi \theta) \subset \partial \Delta$. It is clear that if $R(z)=z^{d}$, for some $d>1$ and $\left.R\right|_{\left\langle x_{i}, x_{i+1}\right\rangle}$ is one-to-one for some $i$, then $\left.f_{X}\right|_{\left\langle x_{i}, x_{i+1}\right\rangle}=\left.R\right|_{\left\langle x_{i}, x_{i+1}\right\rangle}$.

Further we say that a periodic cycle $X=\left(x_{1}, \ldots, x_{n}\right)$ of a continuous endomorphism $R$ of unit circle admits a combinatorial number (or combinatorial rotation number, however this term is already occupied) $\alpha=\frac{p}{n}$, where $\frac{p}{n}$ is in lowest terms, if for any $i=1, \ldots, n$ the interior of the arc $\left\langle x_{i}, R\left(x_{i}\right)\right\rangle$ contains $(p-1)$ points of $X$. It is clear that the linearization of a periodic cycle $X$ is a homeomorphism iff $X$ admits a combinatorial number. In this case $f_{X}$ preserves the natural orientation 
of the unit circle $\partial \Delta$ and is piecewise linear. Hence the map $z \rightarrow|z|^{d} f_{X}(\arg (z))$ is quasiconformal.

Theorem $\mathrm{C}$ is an immediate corollary of Theorem $\mathrm{A}$ and the following theorem.

Theorem 4.1. Let $R(z)=z^{d}, d \geq 2$ and $X=\left(x_{1}<\cdots<x_{n}\right), n>1$ a periodic cycle. Then $X$ has a combinatorial number if and only if there exists a Blaschke map $B$ and a homeomorphism $h: \partial \Delta \rightarrow \partial \Delta$ such that $B=h \circ R(z) \circ h^{-1}$ and the periodic cycle $X^{\prime}=h(X)$ is geodesically accessible from the unit disk $\Delta$.

Proof. The direction " $\Leftarrow$ " is obvious.

Suppose that $X$ has a combinatorial number $\frac{p}{n}$. Then $R\left(x_{i}\right)=x_{i+p}$ for all $i$. Let $f$ be the linearization of $R$ with respect to $X$. Denote by $g$ the map $z \rightarrow$ $|z|^{d} f(\arg (z))$. If $r_{i}$ is a radius going from the point $x_{i}$ to zero, then $g\left(r_{i}\right)=R\left(r_{i}\right)$.

Choose $0<t<1$ and rings $C_{t}^{0}=\left\{z, t^{d} \leq|z| \leq t\right\}$ and $C_{t}^{1}=\left\{z, t^{d^{2}} \leq|z| \leq t^{d}\right\}$. Then $R\left(C_{t}^{0}\right)=g\left(C_{t}^{0}\right)=C_{t}^{1}$. Denote by $\alpha_{i}^{j}$ the segments $r_{i} \cap C_{t}^{j}$. Then $\left.R\right|_{\alpha_{i}^{0}}=\left.g\right|_{\alpha_{i}^{0}}$. Denote by $D_{t}^{j}$ the disk components in $\Delta \backslash C_{t}^{j}$, respectively.

Now, our goal is to construct a branched covering $\mathbf{F}$ from $\Delta$ to $\Delta$ such that

(1) $\mathbf{F}=R$ on the ring component of $\Delta \backslash C_{t}^{0}$ and

(2) $\left.\mathbf{F}\right|_{\left(\partial D_{t}^{0} \cup\left(\bigcup_{i} \alpha_{i}^{0}\right)\right)}=\left.g\right|_{\left(\partial D_{t}^{0} \cup\left(\bigcup_{i} \alpha_{i}^{0}\right)\right)}$.

Construction. Denote by $\Delta_{i}^{j}$ the piece of $C_{T}^{j}$ between $\alpha_{i}^{j}$ and $\alpha_{i+1}^{j}$ in counterclockwise direction.

(1) Set $\mathbf{F}:=R$ on $\Delta \backslash C_{t}^{0}$.

(2) If $R\left(\Delta_{i}^{0}\right)=\Delta_{i+p}^{1}$, then by definition of $\mathrm{g}$ we have $\left.R\right|_{\Delta_{i}^{0}}=\left.g\right|_{\Delta_{i}^{0}}$. In this case we set

$$
\mathbf{F}:=g \text { on the set } \Delta_{i}^{0} .
$$

(3) Let $R\left(\Delta_{i}^{0}\right) \supset \Delta_{i+p}^{1}$ and $R\left(\Delta_{i}^{0}\right) \neq \Delta_{i+p}^{1}$. Then $R\left(\Delta_{i}^{0}\right)=C_{t}^{1}$. Let $n_{i}$ be the degree of the covering $R\left(\Delta_{i}^{0}\right) \rightarrow \Delta_{i+p}^{1}$. Choose any point $z_{i}^{1} \in \Delta_{i+p}^{1}$ and some point $z_{i}^{0} \in R^{-1}\left(z_{i}^{1}\right) \in \Delta_{i}^{0}$. Let $\ell_{i} \subset \Delta_{i}^{1}$ be an arc going from $z_{i}^{1}$ to the point $y_{i}$ of the intersection of $\alpha_{i+p}^{1}$ and the circle $\left\{|z|=t^{d}\right\}$ (see Figure 21). Let $\omega_{i}^{1}<\cdots<\omega_{i}^{n_{i}}$ be the points of $R^{-1}\left(y_{i}\right) \cap \Delta_{i}^{0}$ and let $\ell_{i}^{k} \subset \Delta_{i}^{0}$ be arcs going from the point $z_{i}^{0}$ to $\omega_{i}^{k}$, respectively (see Figure 1). Let $\gamma_{i}^{j} \subset\{|z|=t\}$ be the $\operatorname{arcs}\left\langle\omega_{i}^{k}, \omega_{i}^{k+1}\right\rangle$ and $\gamma_{n_{i}} \subset\{|z|=t\}$ be the $\operatorname{arc}\left\{\Delta_{i}^{0} \cap\{|z|=t\}\right\} \backslash \bigcup_{j}^{n_{i}-1} \gamma_{i}^{j}$.

Consider a partition of $\Delta_{i}^{0}$ and $\Delta_{i}^{1}$ into sets as shown in Figures 1 and 2 Define a map $\mathbf{F}$ on these sets as follows.

(a) Set $\mathbf{F}:=g$ on $\bar{V}$.

(b) Consider W. Let $\Psi$ be a quasiregular branched covering from $W$ onto $W_{1}$ mapping $\ell_{i}^{j}$ onto $\ell_{i}$ such that on any component of $\bar{W} \backslash \bigcup_{j} \bar{\ell}_{i}^{j}$ the map $\Psi$ is one-to-one and $z_{i}^{0}$ is the unique point of branching for $\Psi$ of local degree $n_{i}$ (see Figure 3). Then we set

$$
\mathbf{F}:=\Psi \text { on } \bar{W} .
$$

(c) Consider $U_{k}$. Then $\partial U_{k}=\beta_{k} \cup \gamma_{i}^{k}$, where $\beta_{k} \in \partial W$. The map $\left.\left.R\right|_{\gamma_{i}^{k}} \cup \Psi\right|_{\beta_{k}}$ maps $\partial U_{k}$ onto $\partial W_{1} \cup \partial D_{t}^{0}$. Let $\phi_{k}$ be a map from $\bar{U}_{k}$ onto $D_{t}^{0} \backslash W_{1}$ quasiconformal 


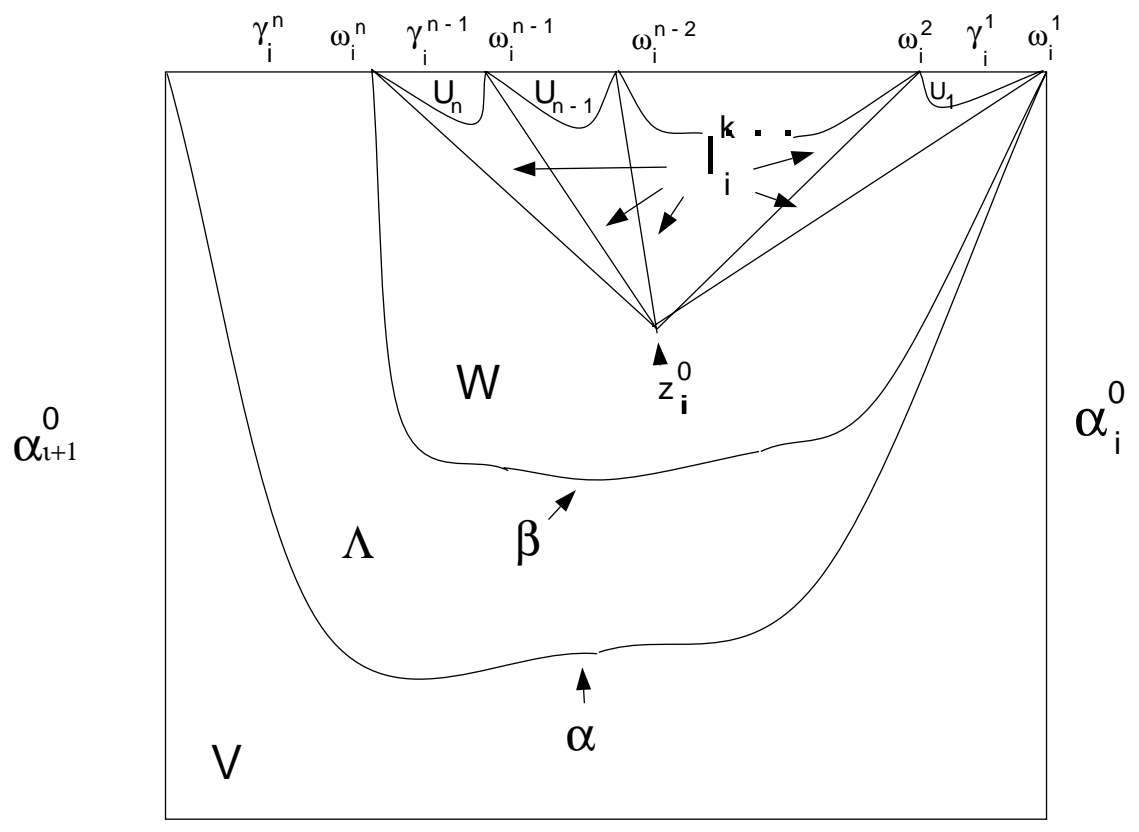

Figure 1. The region $\Delta_{i}^{0}$

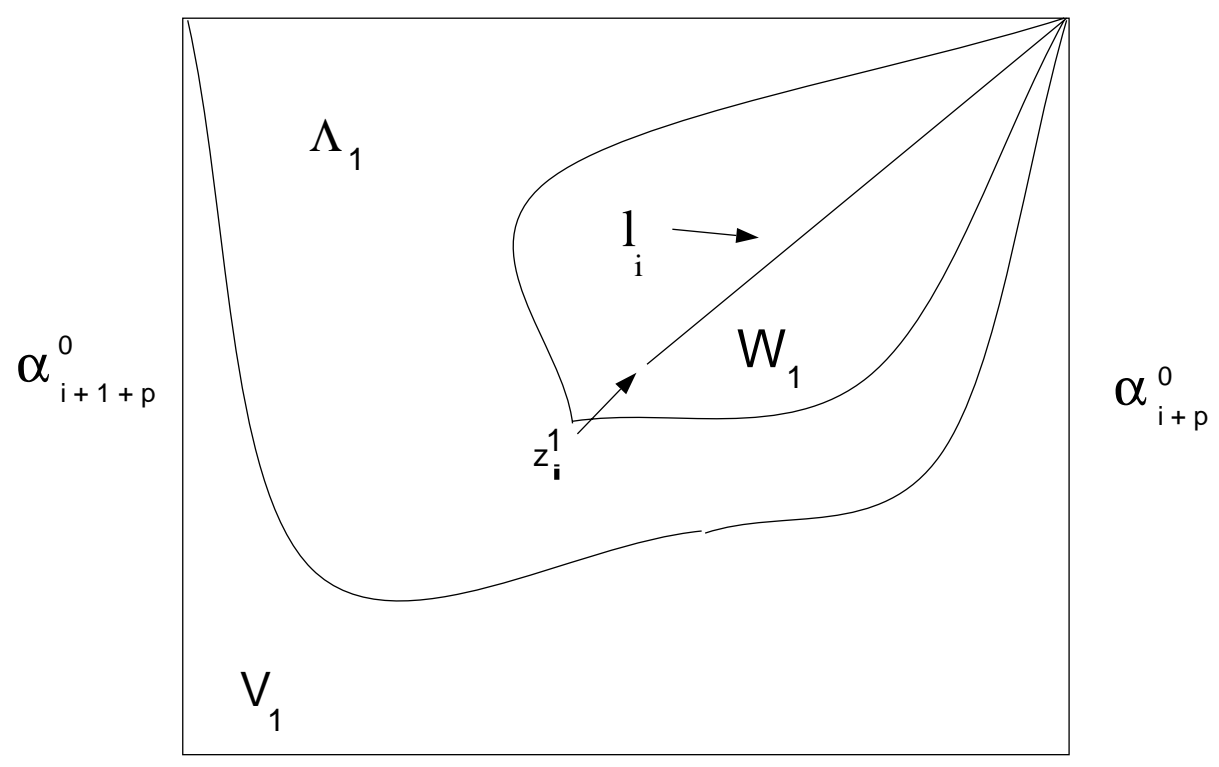

Figure 2. The region $\Delta_{i}^{1}$, here $\bar{V}_{1}=g(\bar{V})$

on the interior of $U_{k}$ and coinciding on $\gamma_{i}^{k}$ and $\beta_{k}$ with $R$ and $\Psi$, respectively. Then we set:

$$
\mathbf{F}:=\phi_{k} \text { on } U_{k}, \text { respectively. }
$$



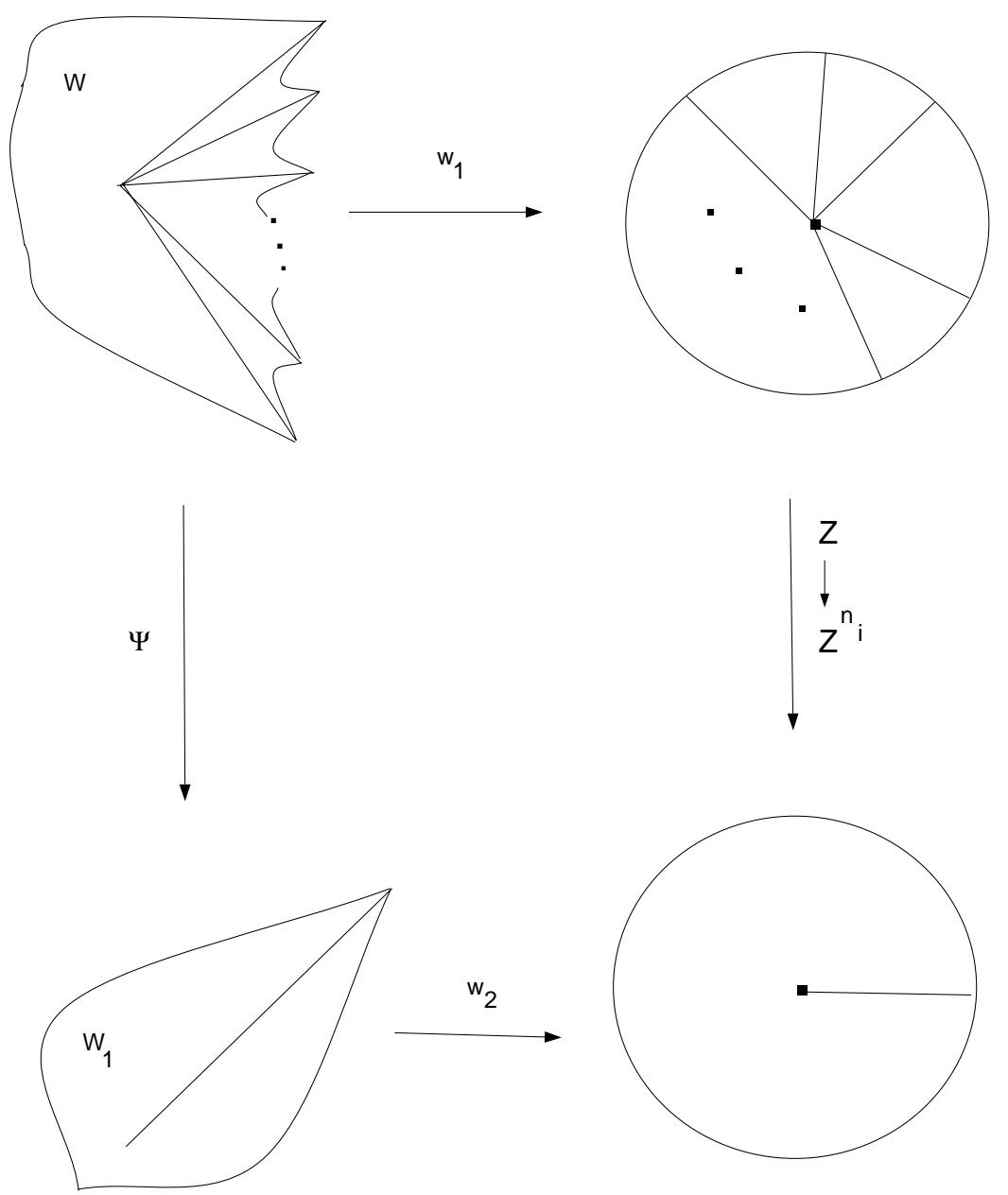

FiguRE 3 . The homeomorphisms $w_{1}$ and $w_{2}$ are quasiconformal

(d) Finally consider $\Lambda$. The boundary of $\Lambda$ consists of $\gamma_{n_{i}} \cup \alpha \cup \beta$, where $\alpha \subset \partial V$ and $\beta \subset \partial W$. The map $\left.\left.\left.R\right|_{\gamma_{k_{i}}} \cup g\right|_{\alpha} \cup \Psi\right|_{\beta}$ maps $\partial \Lambda$ onto $\partial \Lambda_{1}=\partial\left\{\Delta_{i+p}^{1} \backslash W_{1} \backslash V_{1}\right\}$. Let $\Phi$ be a map mapping $\Lambda$ onto $\Lambda_{1}$, coinciding with $R, g$ and $\Psi$ on $\gamma_{k_{i}}$, $\alpha$ and $\beta$, respectively and which is a quasiconformal homeomorphism on the interior of $\Lambda$. Then we set:

$$
\left.\mathbf{F}\right|_{\Lambda}=\Phi
$$

Thus by induction we constructed a map from $\Delta \backslash D_{t}^{0}$ onto $\Delta$. Now consider the ring $C_{t}^{1}$. Then $\mathbf{F}$ is a one-to-one map of the circle $\left\{|z|=t^{d}\right\}$ onto the circle $\left\{|z|=t^{d^{2}}\right\}$. Let $\delta(z)=t^{d} z$ be a map from $D_{t}^{0}$ onto $D_{t}^{1}$ and $H$ be a quasiconformal automorphism of $C_{t}^{1}$ such that

$$
\left.H \circ \mathbf{F} \circ H^{-1}\right|_{\left\{|z|=t^{d}\right\}}=\left.\delta\right|_{\left\{|z|=t^{d}\right\}} .
$$

Then the union $\bigcup_{i}\left\{\bigcup_{n} \delta^{n}\left(H\left(\alpha_{i}^{1}\right)\right)\right\}$ forms $n$ arcs $\beta_{1}, \ldots, \beta_{n}$ such that $H\left(\alpha_{i}^{1}\right) \subset$ $\beta_{i}, \beta_{i} \cap \beta_{j}=\varnothing$ for $i \neq j$ and $\delta\left(\beta_{i}\right)=\beta_{i+p}$. 
Now, we glue $D_{t}^{0}$ with $\delta$ to $\Delta \backslash D_{t}^{1}$ with $\mathbf{F}$ by $\mathrm{H}$ to produce a topological disk $D$ and a branched covering $\Phi: D \rightarrow D$. Construct an invariant conformal structure $\mu$ on $\left\{D_{t}^{0} \cup\left\{\Delta \backslash D_{t}^{1}\right\}\right\} / H$ by $\Phi$ starting with the standard one on $\delta\left(D_{t}^{1}\right)$. Since $\mathbf{F}$ is holomorphic on $\Delta \backslash D_{t}^{0}$ the structure $\mu$ has a bounded distortion. The Measurable Riemann Mapping Theorem gives a conformal map $\phi:(D, \mu) \rightarrow\left(\Delta, \sigma_{0}\right)$, where $\sigma_{0}$ is the standard conformal structure on $\Delta$. Then $B=\phi \circ \Phi \circ \phi^{-1}$ is a Blaschke map and the $\operatorname{arcs} \gamma_{i}=\phi\left(\beta_{i} \cup\left\{z \in r_{i}, r^{d}<|z|\right\}\right)$ go from the attractive point to the periodic cycle $\phi(X)$. By construction the arcs project onto a closed Jordan curve $\gamma^{\prime} \subset S_{\Delta} \subset S_{B}$, and the geodesic $\gamma$ is homotopic to $\gamma^{\prime}$ as desired.

Remark 4.1. In the theorem above we constructed the Blaschke map possibly having critical points of local degree bigger than 2. However, quasiconformal surgery shows that for any structurally stable Blaschke map $B$, any periodic cycle of $B$ having a combinatorial number is geodesically accessible. Thus, in Theorem 4.1 we may take $B$ to be structurally stable.

Now we complete the proof of Theorem [C] Let $B$ be the Blaschke model for $R^{k}: D \rightarrow D$, where $k=L(D)$ and $h$ is the conjugating conformal map. Let $X=\left(x_{1}, \ldots, x_{n}\right) \subset \partial \Delta$ be a periodic point satisfying $\lim _{r \rightarrow 1} h\left(r x_{i}\right)=x$ for any $i$. Then $X$ has a combinatorial number $\frac{m}{n}=\frac{-p}{q}$ or in other words $\frac{m}{n}=\frac{q-p}{q}$, where $\frac{p}{q}$ is the combinatorial rotation number of $x$. Further, the assumption (2) of the theorem and Lemma 1 allow us to assume that $S_{\Delta} \subset S_{B}$ is a $(d-1)$-punctured torus, where $d=d_{i_{0}}$. Therefore Proposition 3.1, Remark 3.1, and Theorem A complete the proof of Theorem $\mathrm{C}$

Corollary C. By Theorem $\mathrm{A}$ one can assume that the Julia set of $R$ is connected.

Assume that all fixed points $x \notin D$ are repelling. Then by a result of Erëmenko and Levin EL all fixed points of $R$ are accessible from the domain $D$. Thus there exists at least one fixed point, say $x$, admitting a non-trivial combinatorial number. Then Lemma 1 and Theorem $\mathrm{C}$ completes this case.

Now, let $x$ be an attractive fixed point and let $D_{1}$ be a corresponding attractive invariant domain. Then $\partial D_{1}$ is a quasicircle. Further, let $Y=\left(y_{1}, y_{2}\right) \subset \partial D_{1}$ be a periodic cycle of period two. Then $Y$ admits the trivial combinatorial rotation number with respect to $D \cup D_{1}$.

Let $B$ and $B_{1}$ be the Blaschke models for $R: D \rightarrow D$ and $R: D_{1} \rightarrow D_{1}$ and $h_{1}$ and $h_{2}$ be the corresponding conformal maps. Then the points $X=\left(x_{1}, x_{2}\right)$ and $X^{\prime}=\left(x_{1}^{\prime}, x_{2}^{\prime}\right) \in \partial \Delta$ satisfying $\lim _{r \rightarrow 1} h_{1}\left(r x_{i}\right)=\lim _{r \rightarrow 1} h_{2}\left(r x_{i}^{\prime}\right)=y_{i}$ are periodic for $B$ and $B_{1}$, respectively and have combinatorial numbers $=\frac{1}{2}$. Then again Theorem $\mathrm{C}$ and Remark 3.1 completes this case together with Corollary $\mathrm{C}$

Remark 4.2. It is easy to see that the above argument also works in the parabolic case except when $\operatorname{deg}\left(\left.R\right|_{D_{1}}\right)=2$.

\section{ACKNOWLEDGEMENT}

I would like to thank the referee for his suggestions and comments, and for his help fixing the language problems. I would like to thank the IMS at SUNY Stony Brook for its hospitality during preparation of this paper.

\section{REFERENCES}

[Ab] L. Ahlfors and L. Bers, Riemann's mapping theorem for variable metrics, Ann. of Math. 72 (1960), 385-401. MR 22:5813 
[DH] A. Douady and J. Hubbard, On the dynamics of polynomial-like mappings, Ann. Scient. École Norm. Sup. 18 (1985), 287-343. MR 87f:58083

[GM] L. R. Goldberg and J. Milnor, Fixed points of polynomials maps, II. Fixed point portraits, Preprint Stony Brook (1990).

[EL] A. Erëmenko and G. M. Levin, Periodic points of polynomials, Ukrainian Math. J. 41 (1989). MR 90m:58102

[L] M. Lyubich, Dynamics of rational transformations: topological picture, Uspekhi Mat. Nauk 41 (1986), 35-95. MR 88g:58094

[Mak] P. Makienko, Pinching and plumbing deformations of quadratic rational maps, Preprint, ICTP 93.32 (1993).

[Mak1] P. Makienko, Unbounded components in parameter space of rational maps, Preprint, ICTP (1993).

[MSS] R. Mañé, P. Sad and D. Sullivan, On the dynamics of rational maps, Ann. Sci. École Norm. Sup. 16 (1983), 193-217. MR 85j:58089

[Mas1] B. Maskit, Comparison of hyperbolic and extremal lengths, Ann. Acad. Sci. Fenn. Ser. A I Math. 10 (1985), 381-386. MR 87c:30062

[Mas2] B. Maskit, Parabolic elements in Kleinian groups, Ann. of Math. 117 (1983), 659-668. MR 85a:30073

[MM1] C. McMullen, Holomorphic Functions and Moduli I, MSRI Publications 10, SpringerVerlag, 1988, pp. 31-60. MR 89m:58187

[MM2] C. McMullen, The classification of conformal dynamical systems, Conference "Current developments in mathematics", Cambridge (May 7-8, 1995). MR 98h:58162

[M] J. Milnor, Remarks on Quadratic maps, Preprint Stony Brook (1992).

[Pil] K. Pilgrim, Ph.D. Thesis, 1994.

[Sh] M. Shishikura, On the quasiconformal surgery of rational functions, Ann. Sci. École Norm. Sup. 20 (1987), 1-29. MR 88i:58099

[Str] K. Strebel, Quadratic differentials, Springer-Verlag, 1984. MR 86a:30072

[S] D. Sullivan, Quasiconformal homeomorphisms and dynamics. I, II, III, Ann. of Math. 2 (1985), 401-418; Acta Math. 155 (1985), 243-260; Adv. Math. 135, 351-395. MR 87i:58103. MR 87i:58104 MR 99e:58145

[Thu] W. P. Thurston, Hyperbolic structures on 3-manifolds I: Deformation of acylindrical manifolds, Ann. of Math. 124 (1986), 203-246. MR 88g:57014

Institute for Applied Mathematics, Shevchenko str. 9, Khabarovsk, 680 000, Russia E-mail address: makienko@iam.khv.ru

Current address: Instituto de Matematicas Unidad Cuernavaca, Universidad Nacional Autonoma de Mexico, A.P. 273-3 Admon. de Correos \#3, 62251 Cuernavaca, Morelos, Mexico

E-mail address: makienko@matcuer.unam.mx 